\title{
Simplicity and Spectrum of Singular Hamiltonian Systems of Arbitrary Order
}

\author{
Huaqing Sun \\ Department of Mathematics, Shandong University at Weihai, Weihai, Shandong 264209, China \\ Correspondence should be addressed to Huaqing Sun; huaqingsun@wh.sdu.edu.cn
}

Received 22 August 2013; Accepted 5 December 2013

Academic Editor: Douglas Anderson

Copyright (C) 2013 Huaqing Sun. This is an open access article distributed under the Creative Commons Attribution License, which permits unrestricted use, distribution, and reproduction in any medium, provided the original work is properly cited.

The paper is concerned with singular Hamiltonian systems of arbitrary order with arbitrary equal defect indices. It is proved that the minimal operator generated by the Hamiltonian system is simple. As a consequence, a sufficient condition is obtained for the continuous spectrum of every self-adjoint extension of the minimal operator to be empty in some interval and for the spectrum to be nowhere dense in this interval in terms of the numbers of linearly independent square integrable solutions.

\section{Introduction}

Consider the following Hamiltonian differential system of arbitrary order $n$ :

$$
\begin{array}{r}
\mathscr{L}(y)(t):=J y^{\prime}(t)-P(t) y(t)=\lambda W(t) y(t), \\
t \in[a, b),
\end{array}
$$

where $a$ is a regular point, while $b$ is singular, that is, $b=+\infty$, or $W$ or $P$ is not integrable near $b ; W(t)$ and $P(t)$ are $n \times n$ Hermitian matrices and locally integrable on $[a, b) ; J$ is an $n \times n$ constant nonsingular matrix satisfying $J^{*}=-J=J^{-1}$ $\left(J^{*}\right.$ denotes the complex conjugate transpose of $\left.J\right) ; W(t) \geq 0$ is a weight function; and $\lambda$ is a complex parameter. System (1) includes higher-order symmetric differential expressions of both even-order and odd-order (cf. [1,2]).

Introduce the following space:

$$
\begin{aligned}
& L_{W}^{2}(a, b) \\
& :=\left\{\text { measurable } f:(a, b) \longrightarrow \mathbf{C}^{n}: \int_{a}^{b} f^{*}(t) W(t) f(t) d t<+\infty\right\},
\end{aligned}
$$

with inner product

$$
\langle f, g\rangle_{W}=\int_{a}^{b} g^{*}(t) W(t) f(t) d t,
$$

for $f, g \in L_{W}^{2}(a, b)$. Set $\|f\|_{W}=\langle f, f\rangle_{W}^{1 / 2}$ for $f \in L_{W}^{2}(a, b)$. We remark that if $W$ is singular, then $L_{W}^{2}(a, b)$ is a quotient space in the sense that $y=z$ if $\|y-z\|_{W}=0$. In this case, $L_{W}^{2}(a, b)$ is a Hilbert space.

Let $\mathrm{AC}_{l}(I)$ denote the set of functions which are locally and absolutely continuous on an interval $I$. In order to ensure the Hamiltonian operators generated by (1) to be singlevalued, it is always assumed that

$$
\begin{gathered}
y \in \mathrm{AC}_{l}((a, b)), \quad f \in L_{W}^{2}(a, b), \quad J y^{\prime}-P y=W f, \\
W y=0 \Longrightarrow y(t)=0 \quad \text { for } t \in[a, b) .
\end{gathered}
$$

Clearly, assumption (A) implies the Atkinson definiteness condition (see [3, Page 253]): for all $\lambda \in \mathbf{C}$ and for all nontrivial solutions $y(t)$ of system (1), the following inequality always holds:

$$
\int_{a}^{b} y^{*}(s) W(s) y(s) d s>0 .
$$


The maximal and preminimal operators $H$ and $H_{00}$ corresponding to system (1) are defined by

$$
\begin{aligned}
& D(H):=\left\{y \in L_{W}^{2}(a, b): y \in \mathrm{AC}_{l}([a, b))\right. \\
& \text { and there exists } f \in L_{W}^{2}(a, b) \\
& \text { such that } \mathscr{L}(y)(t)=W(t) f(t) \text {, } \\
& t \in[a, b)\} \\
& H y:=f \\
& D\left(H_{00}\right) \\
& :=\{y \in D(H): y \text { has a compact support in }(a, b)\} \text {, } \\
& H_{00} y:=H y .
\end{aligned}
$$

It can be easily verified that $H$ is single-valued under assumption (A). The operator $H_{0}:=\bar{H}_{00}$ is called the minimal operator corresponding to system (1).

Spectra of operators have been studied by many authors using various theories such as the oscillation theory, asymptotic analysis, singular sequences, and square-integrable solutions for real values of the spectral parameter (cf., e.g., [413]). Among these methods, the last one has attracted lots of attention because it takes advantage of using numerous tools available in the fundamental theory of differential equations. This method was first explored by Hartman and Wintner for the Sturm-Liouville differential expression

$$
\begin{array}{r}
\tau(x)(t)=-\left(p(t) x^{\prime}(t)\right)^{\prime}+q(t) x(t)=\lambda x(t), \\
t \in[0, \infty),
\end{array}
$$

and a series of results were given (cf. [14-16]). The following is one of them (cf. [15]).

Theorem 1. If $\tau x=\lambda x$ has exactly one linearly independent square integrable solution for all $\lambda$ in an interval $I$, then for every self-adjoint realization, one has the following:

(1) the intersection of continuous spectrum and I is empty;

(2) the point spectrum is nowhere dense in I.

It is well-known that $\tau$ can be classified into the limit point case and the limit circle case at the singular endpoint $t=$ $\infty$, and it is only needed to consider the former case since every self-adjoint realization corresponding to $\tau$ has only a pure discrete spectrum in the limit circle case at $t=\infty$. However, for higher-order differential expressions, there are some intermediate limit cases at $t=\infty$ besides the above two cases (cf. [17]). Weidmann's monograph [13] proved that Theorem 1 holds for higher-order differential equations in the limit point case. This result of [13] was further extended to the case of arbitrary equal defect indices in $[8,12]$. Simple operators in Hilbert spaces have their special properties and have been studied (cf., e.g., [18]). It has been shown that the minimal operators generated by higher-order symmetric differential equations with a regular endpoint are simple (cf., [10, 19]). Recently, Mogilevskii [20] considered simple symmetric operators in Hilbert spaces with equal defect indices and derived that the continuous spectrum of every self-adjoint extension of such an operator is empty in some interval $I$ under the assumption that the number $r(\lambda)$ of linearly independent solutions of an abstract equation is equal to the defect index of the corresponding operator for all $\lambda \in I$ except an at most countable set $X \subset I$ and that the spectrum of every self-adjoint extension of such an operator is nowhere dense in this interval $I$ under the same assumption (see Lemma 4). In addition, these abstract results were applied to higher-order differential operators over arbitrary interval in [20].

In this paper, we will prove that $H_{0}$ with arbitrary equal defect indices is simple inspired by the method in [19] and then apply the above results in [20] to $H_{0}$. As a direct consequence, similar spectral properties of every self-adjoint extension of $H_{0}$ are obtained. Note that we use Mogilevskii's results of [20] to get spectral properties in this paper. Then the method in this paper for the proof of the results on spectral properties of all self-adjoint extensions of $H_{0}$ is different from those used in $[8,12]$.

The rest of this present paper is organized as follows. In Section 2, some basic concepts and results about linear operators in Hilbert spaces are introduced. In Section 3, the simplicity of $H_{0}$ is proved, the results on spectral properties of every self-adjoint extension of $H_{0}$ is given, and two examples are presented.

\section{Basic Concepts and Results about Linear Operators in Hilbert Spaces}

Let $\mathscr{H}$ be a Hilbert space over $\mathbf{C}$ with inner product $\langle\cdot, \cdot\rangle$. The norm $\|\cdot\|$ is $\|f\|=\langle f, f\rangle^{1 / 2}$ for $f \in \mathscr{H}$. Let $T$ be a (linear) operator in $\mathscr{H}$. We denote by $D(T), R(T)$, and $N(T)$ the domain, the range, and the kernel of $T$, respectively, and by $T^{*}$ the adjoint of a densely defined operator $T$. For a densely defined operator $T$, it is called to be symmetric if $T \subset T^{*}$ and self-adjoint if $T=T^{*}$. An operator $S$ is called a self-adjoint extension of a symmetric operator $T$ if $S$ is selfadjoint, $D(T) \subset D(S)$, and $T f=S f$ for $f \in D(T)$.

Let $T$ be an operator in $\mathscr{H}$. If there exists some nontrivial $y \in D(T)$ such that $T y=\lambda y$, then $\lambda$ is called an eigenvalue of $T$, while $y$ is called an eigenvector of $T$ associated with $\lambda$, and $n(T, \lambda)=\operatorname{dim} N(\lambda-T)$ is called the multiplicity of $\lambda$. We denote by $\sigma_{p}(T)$ the set of all eigenvalues of $T$, where $\sigma_{p}(T)$ is called the point spectrum of $T$. Let $R(\lambda, T):=(\lambda-T)^{-1}$. Then, the set

$$
\rho(T):=\{\lambda \in \mathbf{C}: R(\lambda, T)
$$

is a bounded linear operator defined on $\mathscr{H}\}$

is called the resolvent set of $T$, and the set $\sigma(T):=\mathbf{C} \backslash \rho(T)$ is called the spectrum of $T$. When $T$ is a self-adjoint operator, it 
is known in [21, Page 209] that the spectrum $\sigma(T)$ of $T$ admits the representation

$$
\sigma(T)=\overline{\sigma_{p}(T)} \cup \sigma_{c}(T),
$$

where $\sigma_{c}(T)$ is called the continuous spectrum of $T$.

For an operator $T$, the subspace $R(\lambda-T)^{\perp}$ is called the defect space of $\lambda$ and $T$, and the number $\beta(\lambda, T):=\operatorname{dim} R(\lambda-$ $T)^{\perp}$ is called the defect index of $\lambda$ and T. By [21, Theorem $8.1]$, for a symmetric operator $T$, the defect index $\beta(\lambda, T)$ is constant in the upper and lower half-planes, respectively, and hence

$$
d_{+}(T):=\beta(-i, T), \quad d_{-}(T):=\beta(i, T)
$$

are called the positive and negative defect indices of $T$. From [21, Theorem 4.13],

$$
R(\lambda-T)^{\perp}=N\left(\bar{\lambda}-T^{*}\right) .
$$

Hence, $d_{ \pm}(T)=\operatorname{dim} N\left(i \pm T^{*}\right)$. Furthermore, by [21, Theorem 8.6], a symmetric operator $T$ has a self-adjoint extension if and only if

$$
d^{+}(T)=d^{-}(T)=: d(T) .
$$

For a self-adjoint operator, the following is the wellknown spectral decomposition theorem.

Lemma 2 (see [21, Page 191]). For every self-adjoint operator $T$ in $\mathscr{H}$, there exists exactly one spectral family $\{E(t)\}_{t \in \mathbf{R}}$ such that $T=\int_{\mathbf{R}} t d E(t)$. Conversely, the spectral family $\{E(t)\}_{t \in \mathbf{R}}$ is given by

$$
\begin{aligned}
\left\langle g,\left(E\left(t_{2}\right)-E\left(t_{1}\right)\right) f\right\rangle & \\
=(2 \pi i)^{-1} \lim _{\delta \rightarrow 0+} \lim _{\epsilon \rightarrow 0+} \int_{t_{1}+\delta}^{t_{2}+\delta}\langle g,( & R(\sigma-i \epsilon, T) \\
& -R(\sigma+i \epsilon, T)) f\rangle d \sigma
\end{aligned}
$$

for all $f, g \in \mathscr{H}$ and $-\infty<t_{1} \leq t_{2}<\infty$.

Finally, we recall two results for simple symmetric operators in $\mathscr{H}$. A symmetric operator $T$ in $\mathscr{H}$ is called simple if there is not an orthogonal decomposition $T=T_{1} \oplus T_{2}$ with a self-adjoint operator $T_{1}$ acting in a nontrivial subspace $\mathscr{H}_{1} \subset \mathscr{H}$ (cf., [18]). For a simple symmetric operator, the result below holds.

Lemma 3 (see [19]). A symmetric operator $T$ is simple if and only if $f \perp N\left(\lambda-T^{*}\right)$ for all $\lambda \in \mathbf{C} \backslash \mathbf{R}$ implies that $f=0$.

Let $T$ be symmetric and (12) holds. Set

$$
\tilde{\rho}(T)=\left\{\lambda \in \mathbf{R}: \operatorname{dim} N\left(\lambda-T^{*}\right)=d(T)\right\} .
$$

Then, the following result was given.

Lemma 4 (see [20]). Assume that $T$ is a simple symmetric operator in $\mathscr{H}$ and $(12)$ holds with $d(T)<\infty$. Let $I=\left(\mu_{1}, \mu_{2}\right)$, $-\infty \leq \mu_{1}<\mu_{2} \leq \infty$, be an interval such that $I \backslash \tilde{\rho}(T)$ is an at most countable set. Then, for every self-adjoint extension $S$ of $T, \sigma_{c}(S) \cap I$ is empty and $\sigma(S) \cap I$ is nowhere dense in $I$.

\section{Main Results}

With a similar argument to that of [13, Theorem 3.9], the minimal operator $H_{0}$ is symmetric and $H_{0}^{*}=H$. Then, it must have self-adjoint extensions if

$$
d_{+}\left(H_{0}\right)=d_{-}\left(H_{0}\right)=: d .
$$

In this section, we will prove that $H_{0}$ is simple under condition (15) and then get results about the spectrum of every self-adjoint extension of $H_{0}$ using Mogilevskii's results in [20]. First, we present the following lemma.

Lemma 5. Assume that $(A)$ holds and $\lambda \in \mathbf{C} \backslash \mathbf{R}$. Then, there exists an $n \times n$ matrix-valued function $G(t, s, \lambda)$ satisfying

(1) $G(t, s, \lambda)$ is continuous and has first derivative with respect to $t$ on $a<t \leq s<b$ and $a<s \leq t<b$;

(2) $\lim _{t \rightarrow s-0} G(t, s, \lambda)-\lim _{t \rightarrow s+0} G(t, s, \lambda)=J$;

(3) $G$ satisfies that $\mathscr{L} G=\lambda W G$ (as a function of $t$ ) for $t \neq s$;

(4) $G(t, s, \lambda)=G^{*}(s, t, \bar{\lambda})$ for $t \neq s$;

(5) $G(\cdot, s, \lambda) \in L_{W}^{2}(a, b)$, and for $f \in L_{W}^{2}(a, b)$, the function

$$
(\mathscr{G}(\lambda) f)(t):=\int_{a}^{b} G(t, s, \lambda) W(s) f(s) d s
$$

is in $D(H)$ and satisfies that $(\mathscr{L}-\lambda W) \mathscr{G}(\lambda) f=W f$.

Proof. This lemma holds by [22, Formulae (3.23) and (3.24), Lemma 3.8, and Proposition 3.11].

Theorem 6. Assume that (15) holds. Then $\mathrm{H}_{0}$ is simple.

Proof. Since (15) holds, $H_{0}$ must have a self-adjoint extension $H_{1}$. Let $\lambda \in \mathbf{C} \backslash \mathbf{R}$. Then, $R\left(\lambda, H_{1}\right)$ is a bounded operator on $L_{W}^{2}(a, b)$ and for $f \in L_{W}^{2}(a, b)$,

$$
\left(\lambda-H_{1}\right) R\left(\lambda, H_{1}\right) f=f .
$$

Hence, for each $f \in L_{W}^{2}(a, b), R\left(\lambda, H_{1}\right) f \in L_{W}^{2}(a, b)$, and by (5) of Lemma 5,

$$
(\mathscr{L}-\lambda W)\left(R\left(\lambda, H_{1}\right) f+\mathscr{G}(\lambda) f\right)=-W f+W f=0,
$$

which, together with $\mathscr{G}(\lambda) f \in D(H)$, implies that $R\left(\lambda, H_{1}\right) f+$ $\mathscr{G}(\lambda) f \in N(\lambda-H)$. Let $\left\{\theta_{j}(t, \lambda)\right\}_{j=1}^{d}$ be an orthonormal basis for $N(\lambda-H)$. Then

$$
R\left(\lambda, H_{1}\right) f+\mathscr{G}(\lambda) f=\sum_{j=1}^{d} a_{j}(\lambda, f) \theta_{j}(t, \lambda),
$$

where $a_{j}(\lambda, f)$ is a constant dependent on $\lambda$ and $f$ only. It is evident that $R^{*}\left(\lambda, H_{1}\right)=R\left(\bar{\lambda}, H_{1}\right)$ by the self-adjointness of $H_{1}$. Furthermore, $\mathscr{G}(\lambda)$ is an integral operator defined on $L_{W}^{2}(a, b)$ satisfying $\mathscr{G}^{*}(\lambda)=\mathscr{G}(\bar{\lambda})$ by [22, (iii) of Proposition 3.11]. Then, (19) implies that for $j=j=1, \ldots, d$,

$$
\begin{aligned}
a_{j}(\lambda, f) & =\left\langle R\left(\lambda, H_{1}\right) f+\mathscr{G}(\lambda) f, \theta_{j}\right\rangle_{W} \\
& =\left\langle f, R\left(\bar{\lambda}, H_{1}\right) \theta_{j}+\mathscr{G}(\bar{\lambda}) \theta_{j}\right\rangle_{W},
\end{aligned}
$$


while

$$
(\mathscr{L}-\bar{\lambda} W)\left(R\left(\bar{\lambda}, H_{1}\right) \theta_{j}+\mathscr{G}(\bar{\lambda}) \theta_{j}\right)=-W \theta_{j}+W \theta_{j}=0 .
$$

Hence,

$$
\left(R\left(\bar{\lambda}, H_{1}\right)+\mathscr{G}(\bar{\lambda})\right) \theta_{j} \in N(\bar{\lambda}-H) .
$$

Let $\left\{\widetilde{\theta}_{j}(t, \bar{\lambda})\right\}_{j=1}^{d}$ be an orthonormal basis for $N(\bar{\lambda}-H)$. Then, there exist constants $c_{k j}(\lambda)$ dependent on $\lambda$ such that

$$
\left(R\left(\bar{\lambda}, H_{1}\right)+\mathscr{G}(\bar{\lambda})\right) \theta_{j}=\sum_{k=1}^{d} c_{k j}(\lambda) \widetilde{\theta}_{k}, \quad j=1, \ldots, d .
$$

Inserting (23) into (20), we get $a_{j}(\lambda, f)=$ $\sum_{k=1}^{d} \bar{c}_{k j}(\lambda)\left\langle f, \widetilde{\theta}_{k}\right\rangle_{W}$, and then (19) yields that

$$
\begin{aligned}
R\left(\lambda, H_{1}\right) f(t)=- & \int_{a}^{b} G(t, s, \lambda) W(s) f(s) d s \\
& +\sum_{j=1}^{d} \sum_{k=1}^{d} \bar{c}_{k j}(\lambda)\left\langle f, \widetilde{\theta}_{k}\right\rangle_{W} \theta_{j}(t, \lambda) .
\end{aligned}
$$

Now, let $\Phi(t, \lambda)$ and $\Phi(t, \bar{\lambda})$ be fundamental solution matrices for (1) and (1) with $\lambda$ being replaced by $\bar{\lambda}$ satisfying $\Phi(a, \lambda)=\Phi(a, \bar{\lambda})=I_{n}$, respectively, where $I_{n}$ is the $n \times n$ unit matrix. Then,

$$
\Phi^{*}(t, \bar{\lambda}) J \Phi(t, \lambda)=J=-J^{-1},
$$

which implies that $\Phi(t, \lambda) J \Phi^{*}(t, \bar{\lambda})=J$. We define

$$
\begin{aligned}
G_{1}(t, s, \lambda):= & G(t, s, \lambda)+2^{-1} \Phi(t, \lambda) J \Phi^{*}(s, \bar{\lambda}) \\
& + \begin{cases}2^{-1} \Phi(t, \lambda) J \Phi^{*}(s, \bar{\lambda}), & a<s \leq t<b ; \\
-2^{-1} \Phi(t, \lambda) J \Phi^{*}(s, \bar{\lambda}), & a<t<s<b .\end{cases}
\end{aligned}
$$

Then $\mathscr{L} G_{1}=\lambda W G_{1}$ (as a function of $t$ ) for $t \neq s$, and

$$
\begin{aligned}
G(t, s, \lambda)= & G_{1}(t, s, \lambda) \\
& - \begin{cases}\Phi(t, \lambda) J \Phi^{*}(s, \bar{\lambda}), & a<s \leq t<b ; \\
0, & a<t<s<b .\end{cases}
\end{aligned}
$$

Clearly,

$$
\lim _{s \rightarrow t-0} G_{1}^{*}(t, s, \lambda)=\lim _{s \rightarrow t+0} G_{1}^{*}(t, s, \lambda)
$$

by (2) of Lemma 5. Therefore, $G_{1}^{*}(t, \cdot, \lambda) \in \mathrm{AC}_{l}((a, b))$ for any fixed $t \in(a, b)$. On the other hand, $G_{1}^{*}(t, s, \lambda)=G^{*}(t, s, \lambda)$ for $a<t<s<b$ by (27), which, together with (4) and (5) of Lemma 5 , implies that $G_{1}^{*}(t, \cdot, \lambda) \in L_{W}^{2}(c, b)$ for some $c \in$ $(a, b)$. Furthermore,

$$
G_{1}^{*}(t, s, \lambda)=G^{*}(t, s, \lambda)+\Phi(s, \bar{\lambda}) J \Phi^{*}(t, \lambda),
$$

for $a<s \leq t<b$, which, together with $\Phi(\cdot, \bar{\lambda}) \in L_{W}^{2}(a, c)$ by the regularity of the endpoint $a$, implies that $G_{1}^{*}(t, \cdot, \lambda) \in$ $L_{W}^{2}(a, c)$. Thus, $G_{1}^{*}(t, \cdot, \lambda) \in L_{W}^{2}(a, b)$ for every $\lambda \in \mathbf{C} \backslash \mathbf{R}$. In addition, it can be verified that, as a function of $s$,

$$
(\mathscr{L}-\bar{\lambda} W) G_{1}^{*}(t, s, \lambda)=0,
$$

for any fixed $t \in(a, b)$. Consequently, $G_{1}^{*}(t, \cdot, \lambda) \in N(\bar{\lambda}-H)$. Therefore, from (24), (27), and $\widetilde{\theta}_{j} \in N(\bar{\lambda}-H)$, we get that if $f \in N(\lambda-H)^{\perp}$ for all $\lambda \in \mathbf{C} \backslash \mathbf{R}$, then

$$
R\left(\lambda, H_{1}\right) f(t)=\Phi(t, \lambda) J \int_{a}^{t} \Phi^{*}(s, \bar{\lambda}) W(s) f(s) d s
$$

This implies that for $f \in L_{W}^{2}(a, b), R\left(\lambda, H_{1}\right) f$ is a continuous function in $(t, \lambda)$ for $a<t<b$ and all $\lambda \in \mathbf{C}$ since $a$ is regular. Let $\mathscr{C}_{0}$ be a set of $g \in L_{W}^{2}(a, b)$ which has a compact support in $(a, b)$. Then, for $g \in \mathscr{C}_{0}$, the function

$$
\left\langle R\left(\lambda, H_{1}\right) f, g\right\rangle_{W}=\int_{a}^{b} g^{*}(t) W(t) R\left(\lambda, H_{1}\right) f(t) d t
$$

admits an extension to the continuous function in $\mathbf{C}$. This, together with Lemma 2, gives that

$$
\begin{aligned}
& \left\langle g,\left(E\left(t_{2}\right)-E\left(t_{1}\right)\right) f\right\rangle_{W} \\
& =(2 \pi i)^{-1} \lim _{\delta \rightarrow 0+} \lim _{\epsilon \rightarrow 0+} \int_{t_{1}+\delta}^{t_{2}+\delta}\left\langle g,\left(R\left(\sigma+i \epsilon, H_{1}\right)\right.\right. \\
& \left.\left.\quad-R\left(\sigma-i \epsilon, H_{1}\right)\right) f\right\rangle_{W} d \sigma
\end{aligned}
$$

$=0$.

Then, letting $t_{2} \rightarrow+\infty$ and $t_{1} \rightarrow-\infty$, we have $\langle g, f\rangle_{W}=0$, and hence $f=0$ since $\mathscr{C}_{0}$ is dense in $L_{W}^{2}(a, b)$. From Lemma $3, H_{0}$ is simple. This completes the proof.

Let $r(\lambda)$ be the number of linearly independent solutions of (1) in $L_{W}^{2}(a, b)$ and (15) holds. Then, we can get the following from $H=H_{0}^{*}$, Lemma 4, and Theorem 6 .

Theorem 7. Assume that (15) holds. Moreover, let $I=\left(\mu_{1}, \mu_{2}\right)$, $-\infty \leq \mu_{1}<\mu_{2} \leq \infty$, be an interval such that $r(\lambda)=d$ for all $\lambda \in$ Iexcept an at most countable set $X \subset I$. Then, for any selfadjoint extension $H_{1}$ of $H_{0}, \sigma_{c}\left(H_{1}\right) \cap I$ is empty and $\sigma\left(H_{1}\right) \cap I$ is nowhere dense in I.

Example 8. Consider (1) on $[1, \infty)$ with $m=2$, and

$$
\begin{gathered}
J=\left(\begin{array}{cc}
0 & -1 \\
1 & 0
\end{array}\right), \quad P(t)=\left(\begin{array}{cc}
-c & 0 \\
0 & \frac{b}{t^{2}}
\end{array}\right), \\
W(t)=\left(\begin{array}{cc}
1 & 0 \\
0 & \frac{1}{t^{2}}
\end{array}\right),
\end{gathered}
$$


where $b$ and $c$ are real numbers. Then, (1) satisfies assumption (A) since $W(t)>0$ for $t \in[1, \infty)$. Let $\lambda \in \mathbf{R}$. Then, the system has two linearly independent solutions

$$
y_{1}(t)=\left(\begin{array}{c}
(b+\lambda) t^{\omega_{1}} \\
\omega_{1} t^{\omega_{1}+1}
\end{array}\right), \quad y_{2}(t)=\left(\begin{array}{c}
(b+\lambda) t^{\omega_{2}} \\
\omega_{2} t^{\omega_{2}+1}
\end{array}\right),
$$

where $\omega_{1,2}$ are roots of the algebraic equation $\omega^{2}+\omega-(b+$ $\lambda)(c-\lambda)=0$, that is,

$$
\omega_{1,2}=-2^{-1}(1 \pm \sqrt{\Delta}), \quad \Delta=1+4(b+\lambda)(c-\lambda) .
$$

Let $\lambda_{1,2}$ be the roots of $\Delta=0$, that is,

$$
\lambda_{1,2}=2^{-1}\left(c-b \pm \sqrt{(b+c)^{2}+1}\right) .
$$

Then, $\lambda_{1}>\lambda_{2}$ and $\Delta>0$ if $\lambda \in\left(\lambda_{2}, \lambda_{1}\right)$. It can be verified that $\Delta>0$ yields that $y_{1} \in L_{W}^{2}[1, \infty)$ and $y_{2} \notin L_{W}^{2}[1, \infty)$. Thus, if $\lambda \in\left(\lambda_{2}, \lambda_{1}\right)$, then $r(\lambda)=1$. In addition, (15) holds since the coefficients of (1) are real-valued in this case. Furthermore, $1 \leq d \leq 2$ by [17, Theorem 3.3]. If $d=2$, then for all $\lambda \in \mathbf{C}$, all the solutions of $(1)$ are in $L_{W}^{2}[1, \infty)$ by [3, Theorem 9.11.2] in this case. This is a contradiction. Therefore, $d=1$, and then by Theorem 6 , for every self-adjoint extension $H_{1}$ of $H_{0}$ and $I=\left(\lambda_{2}, \lambda_{1}\right), \sigma_{c}\left(H_{1}\right) \cap I$ is empty and $\sigma\left(H_{1}\right) \cap I$ is nowhere dense in $I$.

Consider the $2 \times 2$ Hamiltonian system

$$
\widetilde{J} y^{\prime}(t)+Q(x, \lambda) y(t)=0, \quad t \in\left(t_{0}, b\right],
$$

where $\widetilde{J}=-J$ with $J$ given in Example 8, $Q(t, \lambda)=r(t) A+$ $B(t, \lambda)$ in which $\lambda \in \mathbf{R}, A$ is a constant $2 \times 2$ matrix such that $A=A^{*}$ and $\operatorname{det} A<0$,

$$
\lim _{t \rightarrow t_{0}} \int_{t}^{b} r(s) d s=-\infty, \quad \int_{I_{0}} r(s) d s \leq \rho_{0}
$$

on any subinterval $I_{0} \subset\left(t_{0}, b\right]$ with some constant $\rho_{0}>0$, and $B(\cdot, \lambda)$ is a $2 \times 2$ matrix-valued integrable function on $\left(t_{0}, b\right]$ satisfying that

$$
\int_{t_{0}}^{b}|B(t, \mu)-B(t, \lambda)| d t \longrightarrow 0, \quad \mu \longrightarrow \lambda
$$

Then, by [23, Theorem 3.1], for every $\lambda \in \mathbf{R}$, system (1) has two linearly independent solutions

$$
y_{1}(t)=\left(\begin{array}{l}
h_{11}(t, \lambda) \\
h_{12}(t, \lambda)
\end{array}\right) e^{s(t)}, \quad y_{2}(t)=\left(\begin{array}{l}
h_{21}(t, \lambda) \\
h_{22}(t, \lambda)
\end{array}\right) e^{-s(t)}
$$

where $s(t)=\sigma-\alpha \int_{t}^{b} r(\tau) d \tau$ for some constant $\sigma, \alpha=$ $\sqrt{-\operatorname{det} A}$, and $\lim _{t \rightarrow t_{0}} h_{i j}(t, \lambda)(i, j=1,2)$ exist and are finite for any given $\lambda \in \mathbf{R}$.

Example 9. Consider (1) on $\left(t_{0}, b\right]$ with $m=2$, and

$$
\begin{gathered}
J=\left(\begin{array}{cc}
0 & -1 \\
1 & 0
\end{array}\right), \quad P(t)=\left(\begin{array}{cc}
-\left(t-t_{0}\right)^{-2} & 0 \\
0 & \left(t-t_{0}\right)^{-2}
\end{array}\right), \\
W(t)=\operatorname{diag}\{1,0\} .
\end{gathered}
$$

It can be easily verified that (1) satisfies assumption (A) under the conditions (42). Furthermore, in this case system (1) can be rewritten as (38) with $Q(t, \lambda)=r(t) A+B(t, \lambda), r(t)=$ $-\left(t-t_{0}\right)^{-2}, A=\operatorname{diag}\{1,-1\}$, and $B(t, \lambda)=\operatorname{diag}\{\lambda, 0\}$. It is clear that (39) and (40) hold and $\operatorname{det} A=-1<0$. Therefore, the system has two linearly independent solutions given by (41) with $\alpha=1$. Clearly, $y_{1} \notin L_{W}^{2}\left(t_{0}, b\right)$ and $y_{2} \in L_{W}^{2}\left(t_{0}, b\right)$ for all $\lambda \in \mathbf{R}$. Thus, if $\lambda \in \mathbf{R}$, then $r(\lambda)=1$. Here, let $H_{0}$ be the minimal operator corresponding to this case. With a similar argument to Example 8, it can be obtained that the positive and negative indices of $H_{0}$ are equal to 1 . Note that the corresponding results to Theorem 7 hold for the case that $a$ is singular and $b$ is regular. Then, for every self-adjoint extension $H_{1}$ of $H_{0}, \sigma_{c}\left(H_{1}\right) \cap \mathbf{R}$ is empty and $\sigma\left(H_{1}\right) \cap \mathbf{R}$ is nowhere dense in $\mathbf{R}$.

\section{Conflict of Interests}

The author declares that there is no conflict of interests regarding the publication of this paper.

\section{Acknowledgments}

The author is indebted to the referees for their helpful suggestions and corrections. This research was supported by the NNSFs of China (Grants 11101241 and 11071143), the NNSF of Shandong Province (Grants ZR2011AQ002 and ZR2012AM002), the special fund for postdoctoral innovative programs of Shandong Province (Grant 201301010), and the independent innovation fund of Shandong University (Grant 2011ZRYQ003).

\section{References}

[1] V. I. Kogan and F. S. Rofe-Beketov, "On square-integrable solutions of symmetric systems of differential equations of arbitrary order," Proceedings of the Royal Society of Edinburgh, vol. 74, pp. 5-40, 1976.

[2] P. W. Walker, "A vector-matrix formulation for formally symmetric ordinary differential equations with applications to solutions of integrable square," Journal of the London Mathematical Society, vol. 9, pp. 151-159, 1974/75.

[3] F. V. Atkinson, Discrete and Continuous Boundary Problems, Mathematics in Science and Engineering, Academic Press, New York, NY, USA, 1964.

[4] R. Anderson, "Continuous spectra of a singular symmetric differential operator on a Hilbert space of vector-valued functions," Pacific Journal of Mathematics, vol. 55, pp. 1-7, 1974.

[5] H. Behncke and D. B. Hinton, "Eigenfunctions, deficiency indices and spectra of odd-order differential operators," Proceedings of the London Mathematical Society, vol. 97, no. 2, pp. 425-449, 2008.

[6] H. Behncke and D. Hinton, "Spectral theory of Hamiltonian systems with almost constant coefficients," Journal of Differential Equations, vol. 250, no. 3, pp. 1408-1426, 2011.

[7] H. Behncke and F. O. Nyamwala, "Spectral analysis of higher order differential operators with unbounded coefficients," Mathematische Nachrichten, vol. 285, no. 1, pp. 56-73, 2012.

[8] X. Hao, J. Sun, and A. Zettl, "Real-parameter square-integrable solutions and the spectrum of differential operators," Journal of 
Mathematical Analysis and Applications, vol. 376, no. 2, pp. 696712, 2011.

[9] X. Hao, J. Sun, and A. Zettl, “The spectrum of differential operators and square-integrable solutions," Journal of Functional Analysis, vol. 262, no. 4, pp. 1630-1644, 2012.

[10] C. Remling, "Spectral analysis of higher order differential operators. I. General properties of the $m$-function," Journal of the London Mathematical Society, vol. 58, no. 2, pp. 367-380, 1998.

[11] C. Remling, "Spectral analysis of higher-order differential operators. II. Fourth-order equations," Journal of the London Mathematical Society, vol. 59, no. 1, pp. 188-206, 1999.

[12] J. Sun, A. Wang, and A. Zettl, "Continuous spectrum and square-integrable solutions of differential operators with intermediate deficiency index," Journal of Functional Analysis, vol. 255, no. 11, pp. 3229-3248, 2008.

[13] J. Weidmann, Spectral Theory of Ordinary Differential Operators, vol. 1258 of Lecture Notes in Mathematics, Springer, Berlin, Germany, 1987.

[14] P. Hartman and A. Wintner, "An oscillation theorem for continuous spectra," Proceedings of the National Academy of Sciences of the United States of America, vol. 33, pp. 376-379, 1947.

[15] P. Hartman and A. Wintner, "A separation theorem for continuous spectra," American Journal of Mathematics, vol. 71, pp. 650662, 1949.

[16] P. Hartman and A. Wintner, "On the essential spectra of singular eigenvalue problems," American Journal of Mathematics, vol. 72, pp. 545-552, 1950.

[17] Y. Shi, "On the rank of the matrix radius of the limiting set for a singular linear Hamiltonian system," Linear Algebra and its Applications, vol. 376, pp. 109-123, 2004.

[18] N. I. Akhiezer and I. M. Glazman, Theory of Linear Operators in Hilbert Space II, Pitman, London, UK, 1981.

[19] R. C. Gilbert, "Simplicity of linear ordinary differential operators," Journal of Differential Equations, vol. 11, pp. 672-681, 1972.

[20] V. Mogilevskii, "Symmetric operators with real defect subspaces of the maximal dimension. Applications to differential operators," Journal of Functional Analysis, vol. 261, no. 7, pp. 19551968, 2011.

[21] J. Weidmann, Linear Operators in Hilbert Spaces, vol. 68 of Graduate Texts in Mathematics, Springer, New York, NY, USA, 1980, Translated from the German by Joseph Szücs.

[22] J. Behrndt, S. Hassi, H. de Snoo, and R. Wietsma, "Squareintegrable solutions and Weyl functions for singular canonical systems," Mathematische Nachrichten, vol. 284, no. 11-12, pp. 1334-1384, 2011.

[23] H. Schmid and C. Tretter, "Singular Dirac systems and SturmLiouville problems nonlinear in the spectral parameter," Journal of Differential Equations, vol. 181, no. 2, pp. 511-542, 2002. 


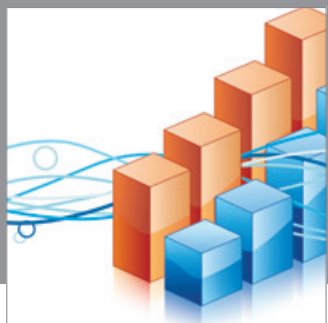

Advances in

Operations Research

mansans

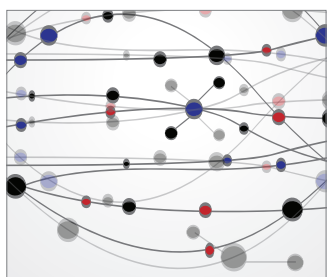

The Scientific World Journal
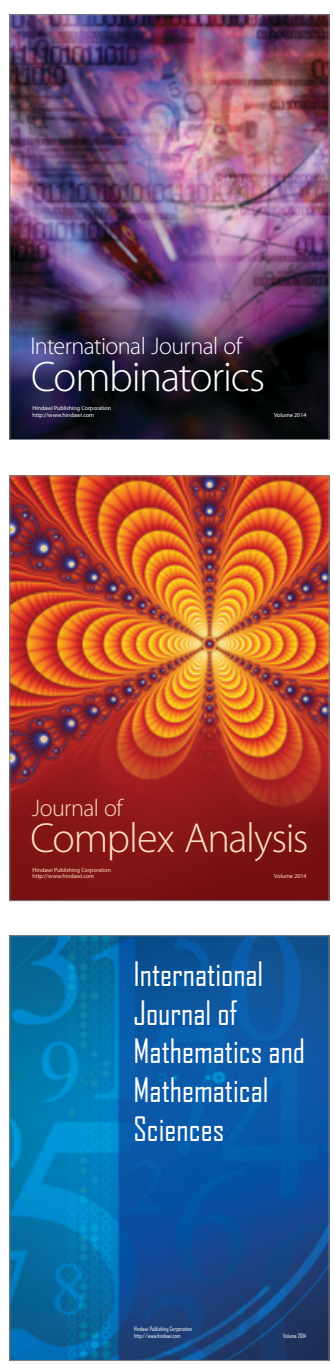
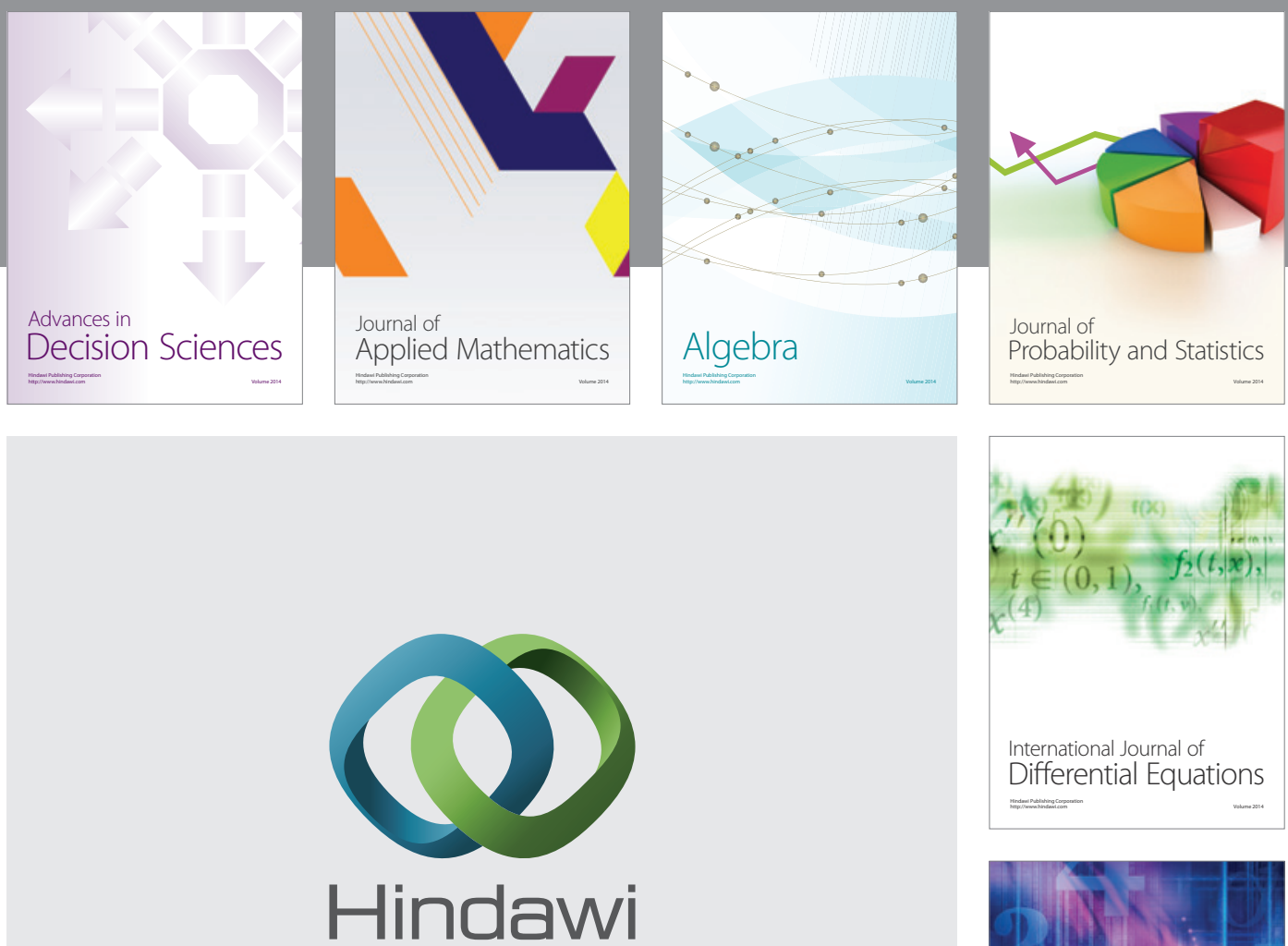

Submit your manuscripts at http://www.hindawi.com
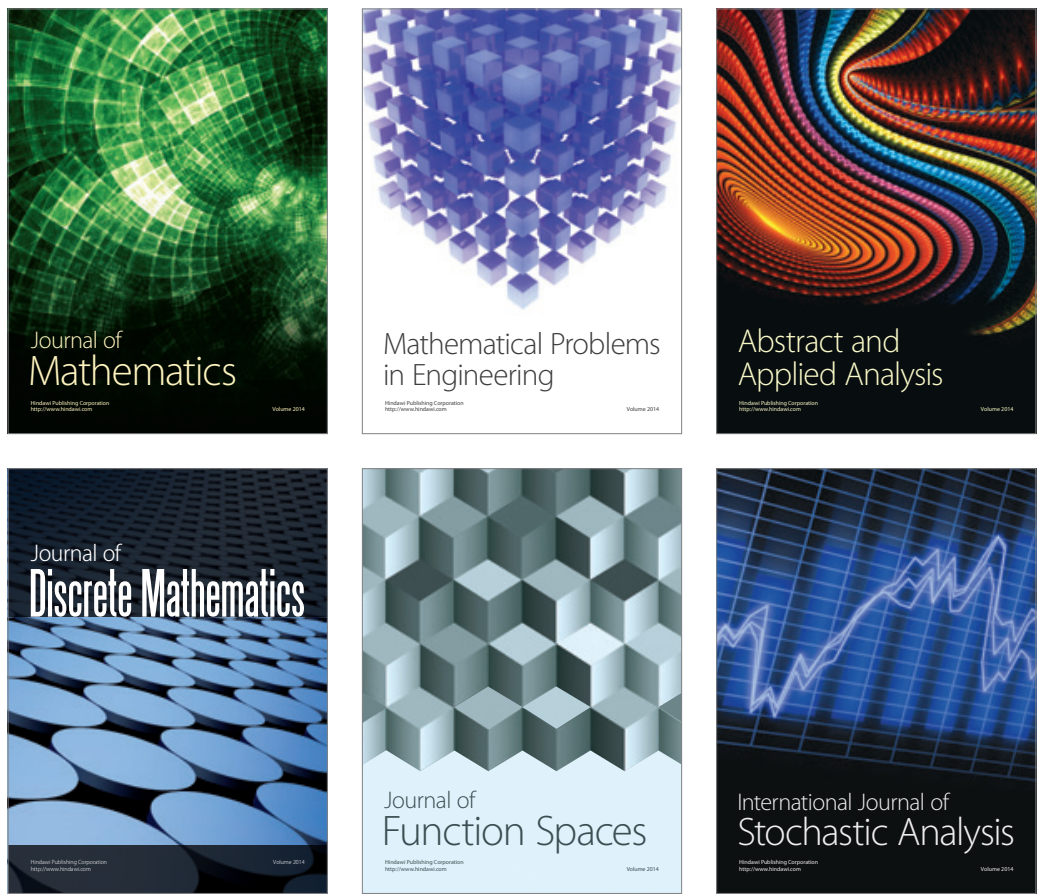

Journal of

Function Spaces

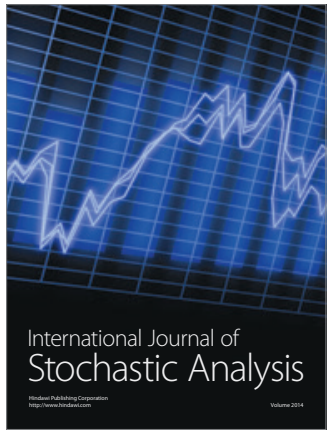

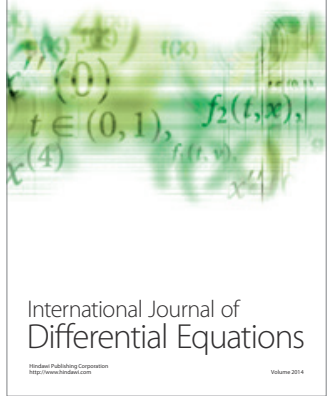
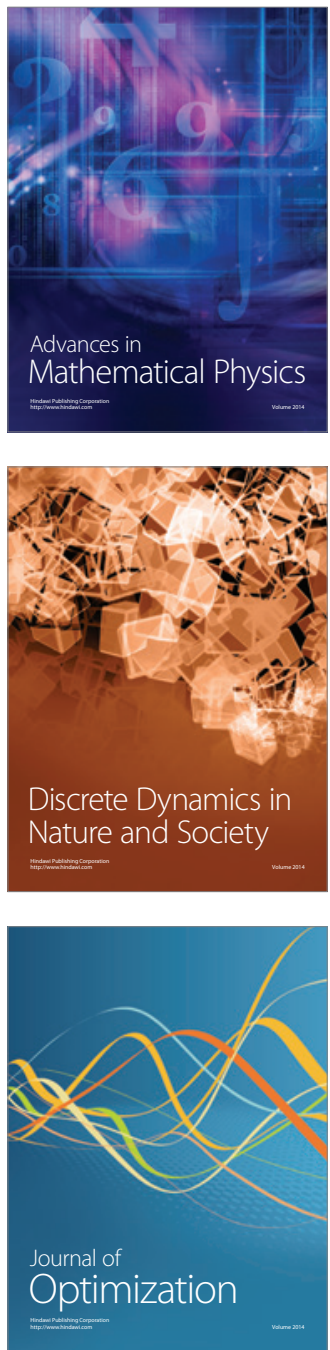